\title{
Theoretical Description of Protein NMR Saturation Transfer to a Bioactive Ligand
}

\author{
N. Lukzen • H. M. Möller
}

\begin{abstract}
Saturation transfer difference nuclear magnetic resonance (STD NMR) is one of the most widely used techniques for detecting and characterizing the interaction of small-molecule ligands with macromolecular receptors. Here, NMR saturation transfer from receptor to ligand and the subsequent propagation within the ligand's nuclear spin system is studied theoretically. We present a consistent treatment of the reversible ligand to protein binding reaction kinetics and extend our analysis to the effect of total correlation spectroscopy (TOCSY) spin locking on spin-to-spin saturation transfer within the ligand. This constitutes a significant improvement of the present theory of the STD NMR method which is based on a rather simplified description of the reversible binding reaction. Furthermore, the dependence of cross-peak intensities in two-dimensional TOCSY spectra on transferred protein NMR saturation to the ligand and the propagation of polarization within the ligand due to strong spin-spin couplings are analyzed. The approach developed here can be generalized to the consideration of other types of nonequilibrium NMR polarization transfer, for instance, chemically induced dynamic nuclear polarization from a protein to its ligand.
\end{abstract}

\section{Introduction}

For many years, nuclear magnetic resonance (NMR) is widely used for research of biological macromolecules and reactions they are involved in. At present, one of the most sensitive and robust techniques for studying ligand-to-protein binding is

\footnotetext{
N. Lukzen

International Tomography Center, Siberian Branch of the Russian Academy of Sciences, Institutskaya st. 3a, 630090 Novosibirsk, Russia

H. M. Möller (ه)

Department of Chemistry, University of Konstanz, 78457 Konstanz, Germany

e-mail: heiko.moeller@uni-konstanz.de
} 
saturation transfer difference (STD) NMR spectroscopy [1-5] (for reviews see Refs. [3-5]).

It has been applied to a vast multitude of receptor-ligand systems, including the recognition of carbohydrates $[6,7]$, peptides [8], small-molecule drugs $[9,10]$, nucleic acid binding compounds [11], with target structures such diverse as proteins, nucleic acids, membrane proteins [12], entire viruses [13], and living cells [14].

Its versatility and robustness are also reflected by its widespread application in ligand screening and lead structure optimization by the pharmaceutical industry [15-17].

In this technique the NMR transitions of a protein are saturated and this saturation is transferred to the ligand when it resides in the binding pocket of the protein receptor [1]. In addition to discriminating between ligands and nonbinding molecules, a detailed epitope mápping of the ligand is possible because the saturation transfer efficiency, i.e., the extent of saturation received by a specific ligand proton depends on its proximity to the protein surface [2]. Combined, this information provides important input to the understanding of biomolecular interactions, their specificity, and to the development of molecules that can interfere with these interactions.

In recent years, a number of alternative techniques and modifications have been developed for the ligand epitope mapping task [18-22] and methods for avoiding complications during epitope mapping that arise from differential relaxation properties of the ligand protons have been introduced [23, 24]. The most sophisticated theoretical treatment of the STD effect, so far, is available through the complete relaxation and conformational exchange matrix-saturation transfer (CORCEMA-ST) approach [25-27]. However, there are still a number of shortcomings associated with the currently available methodology. In the paper we consider two aspects of the STD approach. The first one is a consistent derivation of kinetic equations for the saturation transfer process correctly taking into account the dissociation-association reaction of a ligand-protein complex.

The second aspect is the reconstruction of STD NMR intensities considering the effect of spin locking on the redistribution of STD nuclear polarization within the ligand's nuclear spin system. Individual nuclear polarizations of the ligand's protons are usually easily extracted from one-dimensional (1-D) STD spectra, however, only when there is not too much overlap of the NMR lines. Unfortunately, this is very often the case, especially when studying biomolecular ligands like peptides or oligosaccharides. In the former case, numerous resonances of side chain protons tend to have very similar chemical shifts, e.g., of Lys, Arg, Gln, or Glu. In saccharides, often only the anomeric protons are nicely dispersed, whereas the other ring proton signals are crowded around $4 \mathrm{ppm}$. The 2-D total correlation spectroscopy (TOCSY) technique reduces the overlap problem tremendously, because resonances that fall into the "crowded" regions can be identified and characterized by analyzing TOCSY traces that originate from well-dispersed signals of the same spin system. However, the information content of STD TOCSY spectra has so far only been analyzed semi-quantitatively [8, 29, 30]. In this work we provide a quantitative method for analyzing STD TOCSY spectra. 


\section{Consistent Kinetic Treatment of Ligand Binding in NMR Saturation Transfer}

In Refs. $[25,28]$ it was pointed out that the kinetics of binding can strongly affect the STD amplitudes. Therefore, it is important to give a consistent description for the formation of STD polarization within the ligand. So far, the theoretical treatment of STD NMR in the CORCEMA-ST approach [25] method is based on a rather simplified description of the reversible binding reaction. It describes processes of binding and unbinding in solution by exponential-type kinetics according to the scheme where $\mathrm{P}$ and $L$ stand for the protein and the ligand, respectively, and PL denotes the bound state. The rate constants of association and dissociation are taken as phenomenological ones and their ratio is equal to the equilibrium constant that can be determined experimentally. It was Berg [31] who discovered a "paradox" concerning such scheme of reversible association reaction. At first glance, the decay rate of the ligandprotein complex is equal to its natural (intrinsic) dissociation rate. On the other hand, the association rate, obviously, depends on the mobility (diffusion coefficients) of the reactants. Therefore, the equilibrium constant turns out to depend on mobility as well, which contradicts to the fundamental principle of detailed balance. Berg's [31] detailed analysis of the dissociation and association kinetics resolved this paradox by showing that "a global dissociation as defined from chemical kinetics consists of several molecular dissociations followed by more or less rapid re-associations before diffusion manages to separate the reaction partners". In other words, not every event of the complex dissociation ends up with total diffusion separation of ligand and protein. Since the re-association reaction depends on diffusion, the fraction of globally dissociated complexes also depends on diffusion.

Let us derive kinetic equations reflecting this result of Berg in a formalism of socalled encounter complex formation that takes into account the dissociation reaction, the mutual relative diffusion of reactants (protein and ligand) and the reversibility of the association process. The net reaction scheme in this approach which eliminates the "paradox" is the following: where [P...L] is the so-called encounter complex $[32,33]$. The $\tau_{\mathrm{a}}$ is the characteristic time of protein and ligand association which is occurring from the encounter complex; $\tau_{\mathrm{dis}}$ denotes the dissociation time of the ligand-protein complex (PL). Both $\tau_{\mathrm{a}}$ and $\tau_{\mathrm{dis}}$ are intrinsic constants of the process of ligand and protein association and are therefore viscosity independent. In contrast to that, both rate constants $k_{\mathrm{D}}$ and $k_{\mathrm{d}}$ are viscosity dependent. The former is the usual diffusion-controlled rate $k_{\mathrm{D}}=4 \pi R D$, where $R=R_{\mathrm{L}}+R_{\mathrm{P}}$ is the sum of contact radii of the ligand and the protein and their

$$
\mathrm{P}+\mathrm{L} \rightleftarrows \mathrm{PL},
$$

Scheme 1

$$
\mathrm{P}+\mathrm{L} \underset{k_{\mathrm{d}}}{\stackrel{k_{\mathrm{D}}}{\rightleftarrows}}[\mathrm{P} \ldots \mathrm{L}] \underset{1 / \tau_{\mathrm{dis}}}{\stackrel{1 / \tau_{\mathrm{a}}}{\rightleftarrows}} \mathrm{PL},
$$

Scheme 2 
mutual diffusion coefficient $D=D_{\mathrm{L}}+D_{\mathrm{P}}$ is equal to the sum of their diffusion coefficients $D_{\mathrm{L}}$ and $D_{\mathrm{P}}$. The value $k_{\mathrm{d}}$ can be defined as

$$
k_{\mathrm{d}}=R \Delta / D \text {, }
$$

if we consider that the encounter complex represents itself a thin zone of width $\Delta$ close to the contact $(\Delta<<R)$ [34].

The kinetic equations for concentrations $c_{\mathrm{L}}, c_{\mathrm{P}}, c_{\mathrm{PL}}, c_{[\mathrm{P} \ldots \mathrm{L}]}$ of the ligand, protein, ligand-protein complex, and encounter complex, respectively, in accordance with Scheme (2) are as follows:

$$
\left\{\begin{array}{l}
\frac{\mathrm{d} c_{\mathrm{P}}}{\mathrm{d} t}=-k_{\mathrm{D}} c_{\mathrm{P}} c_{\mathrm{L}}+k_{\mathrm{d}} c_{[\mathrm{P} \ldots \mathrm{L}]} \\
\frac{\mathrm{d} c_{\mathrm{L}}}{\mathrm{d} t}=-k_{\mathrm{D}} c_{\mathrm{P}} c_{\mathrm{L}}+k_{\mathrm{d}} c_{[\mathrm{P} \ldots \mathrm{L}]} \\
\frac{\mathrm{d} c_{[\mathrm{P} \ldots \mathrm{L}]}}{\mathrm{d} t}=k_{\mathrm{D}} c_{\mathrm{P}} c_{\mathrm{L}}-k_{\mathrm{d}} c_{[\mathrm{P} \ldots \mathrm{L}]}+c_{\mathrm{PL}} / \tau_{\mathrm{dis}}-c_{[\mathrm{P} \ldots \mathrm{L}]} / \tau_{\mathrm{a}} \\
\frac{\mathrm{d} c_{\mathrm{PL}}}{\mathrm{d} t}=-c_{\mathrm{PL}} / \tau_{\mathrm{dis}}+c_{[\mathrm{P} \ldots \mathrm{L}]} / \tau_{\mathrm{a}} .
\end{array}\right.
$$

The steady-state approximation for the encounter complex concentration is as follows:

$$
\frac{\mathrm{d} c_{[\mathrm{P} \ldots \mathrm{L}]}}{\mathrm{d} t}=0 .
$$

By taking into account Eqs. (2) and (3) one can express $c_{[\mathrm{P} \ldots \mathrm{L}]}$ through concentrations $c_{\mathrm{L}}, c_{\mathrm{P}}$ and $c_{\mathrm{PL}}$ of the ligand, protein and ligand-protein complex, respectively. Then by substituting the expression into Eq. (2) one obtains the following equations for the concentrations $c_{\mathrm{L}}, c_{\mathrm{P}}$ and $c_{\mathrm{PL}}$ :

$$
\begin{gathered}
\frac{\mathrm{d} c_{\mathrm{L}}}{\mathrm{d} t}=-\frac{k_{\mathrm{D}} / \tau_{\mathrm{a}}}{1 / \tau_{\mathrm{a}}+k_{\mathrm{d}}} c_{\mathrm{L}} c_{\mathrm{P}}+\frac{k_{\mathrm{d}} / \tau_{\mathrm{dis}}}{1 / \tau_{\mathrm{a}}+k_{\mathrm{d}}} c_{\mathrm{PL}}, \\
\frac{\mathrm{d} c_{\mathrm{P}}}{\mathrm{d} t}=-\frac{k_{\mathrm{D}} / \tau_{\mathrm{a}}}{1 / \tau_{\mathrm{a}}+k_{\mathrm{d}}} c_{\mathrm{L}} c_{\mathrm{P}}+\frac{k_{\mathrm{d}} / \tau_{\mathrm{dis}}}{1 / \tau_{\mathrm{a}}+k_{\mathrm{d}}} c_{\mathrm{PL}}, \\
\frac{\mathrm{d} c_{\mathrm{PL}}}{\mathrm{d} t}=\frac{k_{\mathrm{D}} / \tau_{\mathrm{a}}}{1 / \tau_{\mathrm{a}}+k_{\mathrm{d}}} c_{\mathrm{P}} c_{\mathrm{L}}+\frac{k_{\mathrm{d}} / \tau_{\mathrm{dis}}}{1 / \tau_{\mathrm{a}}+k_{\mathrm{d}}} c_{\mathrm{PL}}
\end{gathered}
$$

When introducing the kinetic rate of association

$$
k_{\mathrm{a}}=4 \pi R^{2} \Delta / \tau_{\mathrm{a}},
$$

Eq. (5) can be rewritten in the following form:

$$
\begin{gathered}
\frac{\mathrm{d} c_{\mathrm{L}}}{\mathrm{d} t}=-\frac{k_{\mathrm{D}} k_{\mathrm{a}}}{k_{\mathrm{a}}+k_{\mathrm{D}}} c_{\mathrm{L}} c_{\mathrm{P}}+\frac{1}{\tau_{\mathrm{dis}}} \frac{k_{\mathrm{D}}}{k_{\mathrm{a}}+k_{\mathrm{D}}} c_{\mathrm{PL}}, \\
\frac{\mathrm{d} c_{\mathrm{P}}}{\mathrm{d} t}=-\frac{k_{\mathrm{D}} k_{\mathrm{a}}}{k_{\mathrm{a}}+k_{\mathrm{D}}} c_{\mathrm{L}} c_{\mathrm{P}}+\frac{1}{\tau_{\mathrm{dis}}} \frac{k_{\mathrm{D}}}{k_{\mathrm{a}}+k_{\mathrm{D}}} c_{\mathrm{PL}}, \\
\frac{\mathrm{d} c_{\mathrm{PL}}}{\mathrm{d} t}=\frac{k_{\mathrm{D}} k_{\mathrm{a}}}{k_{\mathrm{a}}+k_{\mathrm{D}}} c_{\mathrm{P}} c_{\mathrm{L}}+\frac{1}{\tau_{\mathrm{dis}}} \frac{k_{\mathrm{D}}}{k_{\mathrm{a}}+k_{\mathrm{D}}} c_{\mathrm{PL}} .
\end{gathered}
$$


One can note from Eq. (6) that the effective dissociation rate constant is viscosity-dependent and is not equal to $1 / \tau_{\mathrm{dis}}$, but is by factor $k_{\mathrm{D}} /\left(k_{\mathrm{D}}+k_{\mathrm{a}}\right)$ smaller. The explanation of this was given by Berg [31] as was mentioned above and is that after the decay of PL to the complex [P...L] only the fraction $k_{\mathrm{D}} /\left(k_{\mathrm{D}}+k_{\mathrm{a}}\right)$ of P and $\mathrm{L}$ "globally" escapes to bulk while the remaining pairs $[\mathrm{P} \ldots \mathrm{L}]$ re-associate to form the ligand-protein complex again. Then one can see that the ratio of rate constants of association and dissociation processes is equal to $k_{\mathrm{a}} \tau_{\text {dis }}$, and now does not depend on viscosity.

The approach described above allows for deriving kinetic equations for concentrations of polarized (saturated) ligand and protein in the free and bound states with consistent account of diffusional motion of the reactants in solution and of their nuclear spin states. To demonstrate this, let us consider the saturation transfer between only two nuclei-one in the protein and another in the ligand. The generalization of this approach to all nuclei, both in the ligand and the protein, is straightforward.

In the quasistationary approximation of the encounter complex [P..L] the following kinetic equations for spin level populations of ligand and protein nuclear spins in their free and bound states can be obtained:

$$
\left\{\begin{array}{l}
\frac{\mathrm{d} c_{\mathrm{L}}^{\dagger}}{\mathrm{d} t}=R_{\mathrm{L}} c_{\mathrm{L}}^{\uparrow}-\frac{k_{\mathrm{a}} k_{\mathrm{D}}}{k_{\mathrm{a}}+k_{\mathrm{D}}} c_{\mathrm{L}}^{\uparrow} c_{\mathrm{P}}+\frac{1}{\tau_{\text {dis }}} \frac{k_{\mathrm{D}}}{k_{\mathrm{a}}+k_{\mathrm{D}}}\left(c_{\mathrm{PL}}^{\uparrow \uparrow}+c_{\mathrm{PL}}^{\uparrow \downarrow}\right), \\
\frac{\mathrm{d} c_{\mathrm{L}}^{\downarrow}}{\mathrm{d} t}=R_{\mathrm{L}} c_{\mathrm{L}}^{\downarrow}-\frac{k_{\mathrm{a}} k_{\mathrm{D}}}{k_{\mathrm{a}}+k_{\mathrm{D}}} c_{\mathrm{L}}^{\downarrow} c_{\mathrm{P}}+\frac{1}{\tau_{\text {dis }}} \frac{k_{\mathrm{D}}}{k_{\mathrm{a}}+k_{\mathrm{D}}}\left(c_{\mathrm{PL}}^{\downarrow \uparrow}+c_{\mathrm{PL}}^{\downarrow \downarrow}\right), \\
\frac{\mathrm{d} c_{\mathrm{P}}^{\dagger}}{\mathrm{d}_{t}}=R_{\mathrm{P}} c_{\mathrm{P}}^{\uparrow}-\frac{k_{\mathrm{a}} k_{\mathrm{D}}}{k_{\mathrm{a}}+k_{\mathrm{D}}} c_{\mathrm{P}}^{\dagger} c_{\mathrm{L}}+\frac{1}{\tau_{\text {dis }}} \frac{k_{\mathrm{D}}}{k_{\mathrm{a}}+k_{\mathrm{D}}}\left(c_{\mathrm{PL}}^{\uparrow \uparrow}+c_{\mathrm{PL}}^{\uparrow \downarrow}\right)-w_{\mathrm{sat}} c_{\mathrm{P}}^{\uparrow}+w_{s a t} c_{\mathrm{P}}^{\downarrow}, \\
\frac{\mathrm{d} c_{\mathrm{P}}^{\downarrow}}{\mathrm{d} t}=R_{\mathrm{P}} c_{\mathrm{P}}^{\downarrow}-\frac{k_{\mathrm{a}} k_{\mathrm{D}}}{k_{\mathrm{a}}+k_{\mathrm{D}}} c_{\mathrm{P}}^{\downarrow} c_{\mathrm{L}}+\frac{1}{\tau_{\text {dis }}} \frac{k_{\mathrm{D}}}{k_{\mathrm{a}}+k_{\mathrm{D}}}\left(c_{\mathrm{PL}}^{\uparrow \downarrow}+c_{\mathrm{PL}}^{\downarrow \downarrow}\right)-w_{\mathrm{sat}} c_{\mathrm{P}}^{\downarrow}+w_{s a t} c_{\mathrm{P}}^{\uparrow},
\end{array}\right.
$$

and for the ligand-protein complex:

$$
\frac{\mathrm{d}}{\mathrm{d} t}\left(\begin{array}{c}
c_{\mathrm{PL}}^{\uparrow \uparrow} \\
c_{\mathrm{PL}}^{\uparrow \downarrow} \\
c_{\mathrm{PL}}^{\uparrow \uparrow} \\
c_{\mathrm{PL}}^{\downarrow \downarrow}
\end{array}\right)=W_{\mathrm{NOE}}\left(\begin{array}{c}
c_{\mathrm{PL}}^{\uparrow \uparrow} \\
c_{\mathrm{PL}}^{\uparrow \downarrow} \\
c_{\mathrm{PL}}^{\uparrow \uparrow} \\
c_{\mathrm{PL}}^{\downarrow \downarrow}
\end{array}\right)+\frac{k_{\mathrm{a}} k_{\mathrm{D}}}{k_{\mathrm{a}}+k_{\mathrm{D}}}\left(\begin{array}{c}
c_{\mathrm{P}}^{\uparrow} c_{\mathrm{L}}^{\uparrow} \\
c_{\mathrm{P}}^{\uparrow} c_{\mathrm{L}}^{\downarrow} \\
c_{\mathrm{P}}^{\downarrow} c_{\mathrm{L}}^{\uparrow} \\
c_{\mathrm{P}}^{\downarrow} c_{\mathrm{L}}^{\downarrow}
\end{array}\right)-\frac{1}{\tau_{\mathrm{dis}}} \frac{k_{\mathrm{D}}}{k_{\mathrm{a}}+k_{\mathrm{D}}}\left(\begin{array}{c}
c_{\mathrm{PL}}^{\uparrow \uparrow} \\
c_{\mathrm{PL}}^{\uparrow \downarrow} \\
c_{\mathrm{PL}}^{\uparrow \uparrow} \\
c_{\mathrm{PL}}^{\downarrow \downarrow}
\end{array}\right) .
$$

Here indices "P", "L", and "PL" stand for the protein, ligand, and bound state, respectively, arrows indicate projection of nuclear spin (spins), " $\uparrow "$ for $+1 / 2$ and " $\downarrow$ " for $-1 / 2$. The quantities

$$
C_{\mathrm{L}}^{\uparrow}, C_{\mathrm{L}}^{\downarrow}, C_{\mathrm{P}}^{\uparrow}, C_{\mathrm{P}}^{\downarrow}, C_{\mathrm{PL}}^{\uparrow \downarrow}, C_{\mathrm{PL}}^{\downarrow \uparrow}, C_{\mathrm{PL}}^{\uparrow \uparrow}, C_{\mathrm{PL}}^{\downarrow \downarrow}
$$

denote concentrations of corresponding species in different spin states. Operators $R_{\mathrm{L}}, R_{\mathrm{P}}$, and $W_{\mathrm{NOE}}$ describe spin relaxation of protein and ligand, and bound state nuclei due to $T_{1}$ and nuclear Overhauser effect. The quantity $w_{\text {sat }}$ is the rate of radiofrequency saturation of protein nuclei.

In this derivation we used formal procedure elaborated in our recent paper [33] where we formalize the derivation of kinetic equations for any number of reactants taking part in bimolecular reactions:

$$
A_{i}+B_{j} \rightleftarrows A_{k}+B_{l}, \quad i, k=1, \ldots K, \quad j, l=1, \ldots, L,
$$

and in association-dissociation reactions: 


$$
A_{i}+B_{j} \rightleftarrows C_{m}, \quad m=1, \ldots, M .
$$

In our case a set of reactants of type $A$ are two ligands with $(+1 / 2)$ and $(-1 / 2)$ projection of the nuclear spin, respectively. Two reactants of type $B$ are proteins with $+1 / 2$ and $-1 / 2$ projections of the nuclear spin, respectively, while four reactants of type $\mathrm{C}$ are ligand-protein complexes with different projections of ligand and protein nuclear spins. The main result obtained in Ref. [33] is the formalization of kinetic equation derivation for reactants and reaction schemes Eqs. (9) and (10). For instance, for reactants of type A the following kinetic equation was obtained:

$$
\frac{\mathrm{d} N_{A_{i}}}{\mathrm{~d} t}=\sum_{j, k, l}\left\langle A_{i} B_{j}\left|\hat{R}_{A B}\right| A_{k} B_{l}\right\rangle N_{A_{k}} N_{B_{l}}+\sum_{j, m}\left\langle A_{i} B_{j}\left|\hat{R}_{d}\right| C_{m}\right\rangle N_{C_{m}}
$$

where $N_{A_{i}}$ and $N_{B_{j}}$ are concentrations of the species $A_{i}$ and $B_{j}$, respectively.

Matrix operators $\widehat{R}_{A B}$ and $\widehat{R}_{d}$ in Eq. (11) are defined as

$$
\hat{R}_{A B}=\left(\widehat{E}-\widehat{K}_{A B} \widehat{G}\right)^{-1} \hat{K}_{A B}, \quad \hat{R}_{d}=\left(\hat{E}-\widehat{K}_{A B} \widehat{G}\right)^{-1} \hat{K}_{d} .
$$

The operators $\widehat{K}_{A B}, \widehat{K}_{d}$ are constructed from rates $k_{\mathrm{a}}, k_{\mathrm{D}}, \tau_{\mathrm{dis}}$ in a very simple and clear manner (see equations (3.16)-(3.20) in Ref. [33]). Finally, one has to add in kinetic equation terms which describe NOE and spin relaxation contribution and then one arrives at Eqs. (7) and (8).

Equations (7) and (8) can be reduced to ones for nuclear spin polarization of a free ligand $\left(M_{\mathrm{L}}^{z}\right)$, a free protein $\left(M_{\mathrm{P}}{ }^{z}\right)$ and their polarizations in binding states $\left(I_{\mathrm{L}}{ }^{z}\right.$, $\left.I_{\mathrm{P}}{ }^{z}\right)$ :

$$
\left\{\begin{array}{l}
\frac{\mathrm{d} M_{\mathrm{L}}^{z}}{\mathrm{~d} t}=-\frac{1}{T_{1 \mathrm{~L}}}\left(M_{\mathrm{L}}^{z}-M_{0 \mathrm{~L}}\right)-\frac{k_{\mathrm{a}} k_{\mathrm{D}}}{k_{\mathrm{a}}+k_{\mathrm{D}}} M_{\mathrm{L}}^{z} c_{\mathrm{P}}+\frac{1}{\tau_{\mathrm{dis}}} \frac{k_{\mathrm{D}}}{k_{\mathrm{a}}+k_{\mathrm{D}}} I_{\mathrm{L}}^{z}, \\
\frac{\mathrm{d} M_{\mathrm{P}}^{z}}{\mathrm{~d} t}=-\frac{1}{T_{\mathrm{IP}}}\left(M_{\mathrm{P}}^{z}-M_{0 \mathrm{P}}\right)-\frac{k_{\mathrm{a}} k_{\mathrm{D}}}{k_{\mathrm{a}}+k_{\mathrm{D}}} M_{\mathrm{P}}^{z} c_{\mathrm{L}}+\frac{1}{\tau_{\mathrm{dis}}} \frac{k_{\mathrm{D}}}{k_{\mathrm{a}}+k_{\mathrm{D}}} I_{\mathrm{P}}^{z}-2 w_{\mathrm{sa}} M_{\mathrm{P}}^{z} \\
\frac{\mathrm{d}}{\mathrm{d} t}\left(\begin{array}{c}
I_{\mathrm{L}}^{z} \\
I_{\mathrm{P}}^{z}
\end{array}\right)=\left(\begin{array}{l}
-\rho_{\mathrm{L}}-\sigma_{\mathrm{PL}} \\
-\rho_{\mathrm{P}}-\sigma_{\mathrm{LP}}
\end{array}\right)\left(\begin{array}{l}
I_{\mathrm{L}}^{z}-M_{0 \mathrm{~L}} \\
I_{\mathrm{P}}^{z}-M_{0 \mathrm{P}}
\end{array}\right)+\frac{k_{\mathrm{a}} k_{\mathrm{D}}}{k_{\mathrm{a}}+k_{\mathrm{D}}}\left(\begin{array}{l}
M_{\mathrm{L}}^{z} c_{\mathrm{P}} \\
M_{\mathrm{P}}^{z} c_{\mathrm{L}}
\end{array}\right)-\frac{1}{\tau_{\mathrm{dis}}} \frac{k_{\mathrm{D}}}{k_{\mathrm{a}}+k_{\mathrm{D}}}\left(\begin{array}{c}
I_{\mathrm{L}}^{z} \\
I_{\mathrm{P}}^{z}
\end{array}\right) \\
I_{\mathrm{L}}^{z}=c_{\mathrm{PL}}^{\uparrow \uparrow}-c_{\mathrm{PL}}^{\uparrow \downarrow}+c_{\mathrm{PL}}^{\downarrow \uparrow}-c_{\mathrm{PL}}^{\downarrow \downarrow} ; I_{\mathrm{P}}^{z}=c_{\mathrm{PL}}^{\uparrow \uparrow}+c_{\mathrm{PL}}^{\uparrow \downarrow}-c_{\mathrm{PL}}^{\downarrow \uparrow}-c_{\mathrm{PL}}^{\downarrow l}
\end{array}\right.
$$

Here $M_{\mathrm{OP}}$ and $M_{\mathrm{OL}}$ are equilibrium polarizations of protein and ligand, respectively, in the magnetic field of the NMR spectrometer.

Kinetic equations for the ligand and protein concentrations

$$
c_{\mathrm{L}}=c_{\mathrm{L}}^{\downarrow}+c_{\mathrm{L}}^{\uparrow}, c_{\mathrm{P}}=c_{\mathrm{P}}^{\downarrow}+c_{\mathrm{P}}^{\uparrow}
$$

are the same as in Eq. (6).

These equations allow for determining the degree of saturation transfer for any pulse sequence, for variable extent of protein saturation, its dependence on ligand and protein concentrations, viscosity of the media and hence to determine the optimal experimental conditions for STD observation.

Note that it is known that the statistics of recontacts of reactants during their encounter in liquid is substantially nonexponential. But it is known [33] that 
Scheme (2) which implies an exponential decay of the encounter complex is still correct when determining the reaction rate constants. Reactants in liquid experience many recontacts during encounter; thus the processes of binding and unbinding can proceed many times within the same pair of the reactants. This can result in a qualitatively different dependence of the transferred polarization amplitudes on viscosity and unbinding rate constant than that given by Scheme (1). Moreover, even using Scheme (2) one may draw wrong conclusions about the degree of proximity of different protons to the protein in the binding PL state in the case of a flexible ligand whose conformation changes between recontacts such that subsequent binding occurs with this new conformation of the ligand or when subsequent binding events occur with another orientation of the (even rigid) ligand. In these cases (though this should be quite unlikely in the vast majority of biomolecular interactions) an even more comprehensive/complicated treatment has to be employed to achieve a consistent description of diffusion-controlled reactions outlined in a set of papers [35-38].

The equations obtained can be employed also for analysis of protein chemically induced dynamic nuclear polarization (CIDNP) transfer to the ligand. It is well known that few amino acids are CIDNP active. In the case when a protein binding pocket contains some of these amino acids CIDNP can be created in course of a photochemical reaction with the excited dye on the amino acids. The CIDNP can be transferred then onto the ligand during binding. To describe this one has to add equations of CIDNP creation due to photoreaction of the excited dye with CIDNPactive amino acids of the protein pocket. The supposed advantage of such way of studying ligand-protein binding lies in the fact that the CIDNP signal usually significantly exceeds the equilibrium nuclear polarization and therefore one could expect that detection by this way will be much more sensitive than by STD NMR. But considering this approach one has to analyze the interplay between the process of CIDNP formation and ligand binding. On the one hand the binding pocket of the protein should be free from the ligand, for the dye could reach the CIDNP-active amino acids. This implies that the concentration of ligands should be less or equal to the protein concentration. On the other hand, one needs a higher concentration of ligands to increase the frequency of encounters with CIDNP-polarized proteins to provide efficient CIDNP transfer within the $T_{1}$ relaxation time of the amino acids protons.

\section{Determination of the Initial Saturation of Ligand Nuclei from 2-D STD TOCSY Spectra}

To correctly determine the degree of spatial proximity to the protein of various protons of the ligand from the STD TOCSY spectrum, one must take into account the redistribution of transferred NMR saturation within the nuclear spin system of the ligand during the TOCSY spin-locking stage. This redistribution is brought about by the TOCSY spin lock field that imposes the strong coupling regime to all scalar coupled spin pairs. Therefore, to interpret TOCSY spectra with the purpose to reconstruct the initial polarization transferred from the saturated protein to its 
Table 1 Chemical shifts and spin-spin coupling constants $J$ used in calculations

\begin{tabular}{llllllll}
\hline Proton & $\mathrm{H} 1$ & $\mathrm{H} 2$ & $\mathrm{H} 3$ & $\mathrm{H} 4$ & $\mathrm{H} 5$ & $\mathrm{H} 6$ & H7 \\
\hline Chemical shift (ppm) & 4.590 & 3.751 & 3.569 & 3.480 & 3.492 & 3.733 & 3.920 \\
Protons & $\mathrm{H} 1 / \mathrm{H} 2$ & $\mathrm{H} 2 / \mathrm{H} 3$ & $\mathrm{H} 3 / \mathrm{H} 4$ & $\mathrm{H} 4 / \mathrm{H} 5$ & $\mathrm{H} 5 / \mathrm{H} 6$ & $\mathrm{H} 5 / \mathrm{H} 7$ & H6/H7 \\
$J(\mathrm{~Hz})$ & 8.4 & 10.1 & 8.2 & 9.3 & 5.5 & 2.7 & 12.4 \\
\hline
\end{tabular}

ligand, it is necessary to study the coherent transfer of nonequilibrium polarization between strongly spin-spin coupled nuclei.

As a model system we chose the non-reducing $\mathrm{N}$-acetylglucosamine (GlcNAc) residue of chitobiose measured in $\mathrm{D}_{2} \mathrm{O}$. This molecular fragment is present in all $\mathrm{N}$-glycosylated glycopeptides as well as in many naturally occurring oligosaccharides. Only the ring protons and the 6,6'-methylene protons were considered here. The methyl group of the $\mathrm{N}$-acetyl residue is separated from the ring spin system, and the amide proton is exchanged into deuterium in $\mathrm{D}_{2} \mathrm{O}$ solution. With its spin-system comprising of 7 protons GlcNAc is a good model for the majority of monosaccharide building blocks and also for many amino acid residues. Chemical shifts and $J$-couplings are given in Table 1 .

A computer program that mimics the acquisition of 2-D TOCSY spectra was written and is available from the authors upon request. The output format was adjusted for visualization and analysis within the MestRe-C/MestreNOVA software (Mestrelab Research, Santiago de Compostela, Spain, http://www.mestrelab.com). This allows, for instance, easy determination of 2-D cross-peak integrals and will make our simulation program intuitively useful to the MestreNOVA community.

The program can be used for simulating the STD TOCSY spectra, as well as for calculating the CIDNP-TOCSY spectra of ligands like saccharides or peptides. It can easily be extended to handle spin systems with more than seven spins and could then be applied to virtually all relevant types of ligand structures. In the case of peptides and oligosaccharides it is of course advantageous that the spin systems of each building block are not connected by scalar couplings and can thus be subjected to the simulation procedure separately which significantly speeds up the necessary calculations. As an example, Fig. 1 represents the TOCSY spectrum of all protons being in thermal equilibrium, as calculated by our program. There are totally 25 cross and diagonal peaks in the spectrum. Our approach is designed for reconstructing the initial transferred saturation onto individual nuclei of the ligand from peak integrals of the detected 2-D STD TOCSY spectrum. It turns out that such a spectrum can be constructed from a linear combination of a set of "subspectra", each of which has been calculated with all but one nucleus saturated since the STD (and NOE) process does not give rise to any multiplet nuclear polarization. The initial density matrix $\sigma$ of the ligand then has to be taken in the following form:

$$
\sigma=\alpha E+\sum_{i} w_{i} \hat{I}_{i z}
$$

where $\widetilde{I}_{i z}$ is the operator of $z$-projection of the $i$-th spin, $\alpha$ is a coefficient, $E$ is a unity 


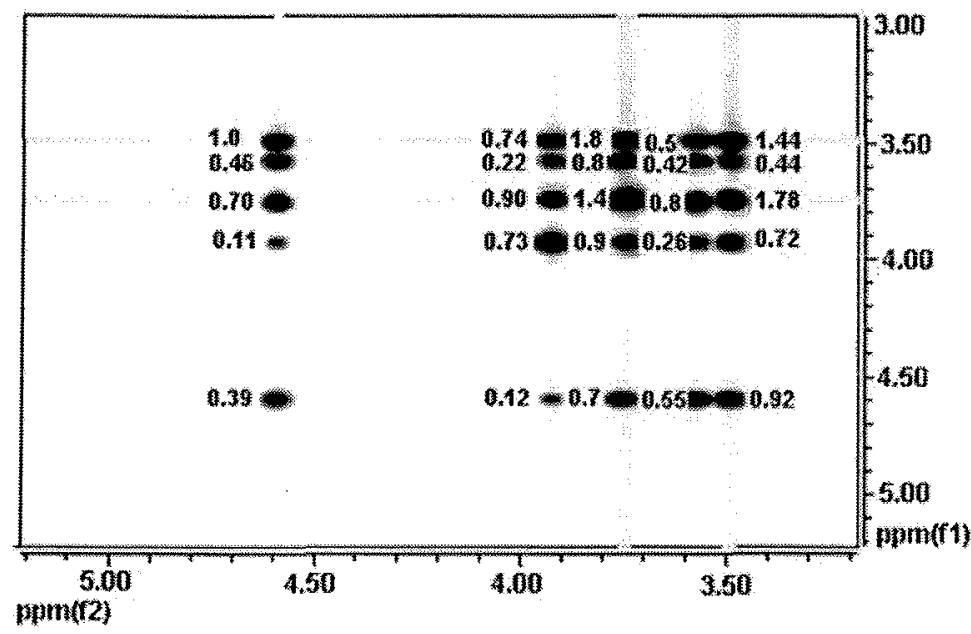

Fig. 1 Calculated TOCSY spectrum of all protons carrying equilibrium polarization. Next to peaks their integrals are indicated

matrix, and the trace of the $\alpha E$ matrix is equal to 1 . Applying the chemical shifts of the GlcNAc ring protons, five clearly distinguishable peak positions are obtained leading to, in total, 25 cross- and diagonal peaks in the TOCSY spectrum. The sets of integrals of these 25 peaks (the peak positions are indicated in Fig. 1) were calculated for seven vectors of the type

$$
w=\left\{\delta_{i k}\right\}(k=1 \ldots 7),
$$

each of them corresponding to saturation of all spins except the $i$-th one.

This means that the corresponding density matrix $\sigma_{i}$ was taken as follows:

$$
\sigma_{i}=\alpha E+I_{i z}
$$

Thus, $7 \times 25$ integrals were collected and the matrix $S$ of dimension $7 \times 25$ containing these integrals was constructed. The treatment of experimental data, i.e., finding values $w_{i}$ from peak integrals of the experimental TOCSY spectrum, is then very simple. The values $w_{i}$ can be found as follows:

$$
\vec{w}=S^{-1} \vec{I}_{\text {exp }}
$$

where $\vec{I}_{\text {exp }}$ is a column of 25 integrals of cross and diagonal peaks of the experimental TOCSY spectrum. This allows one to extract the saturation (polarization) of each ligand nucleus transferred from protein from the TOCSY spectrum.

For the purpose of testing our approach we conducted model calculations. We calculated by our program TOCSY spectra for weights $w_{1}=(0,0.7,0,0,0,0,0)$ (Fig. 2) and $w_{2}=(0,0.7,0,0,0,1.0,0)$ (Fig. 3) that correspond to the polarized second or the second and the sixth nuclei, respectively, while other nuclei have zero polarization. Even though the second and the sixth nuclei have very similar chemical shifts (3.751 and $3.733 \mathrm{ppm}$ ) and are therefore virtually indistinguishable in 1-D as well as in 2-D TOCSY spectra, our inverse procedure (Eq. 15) employing 


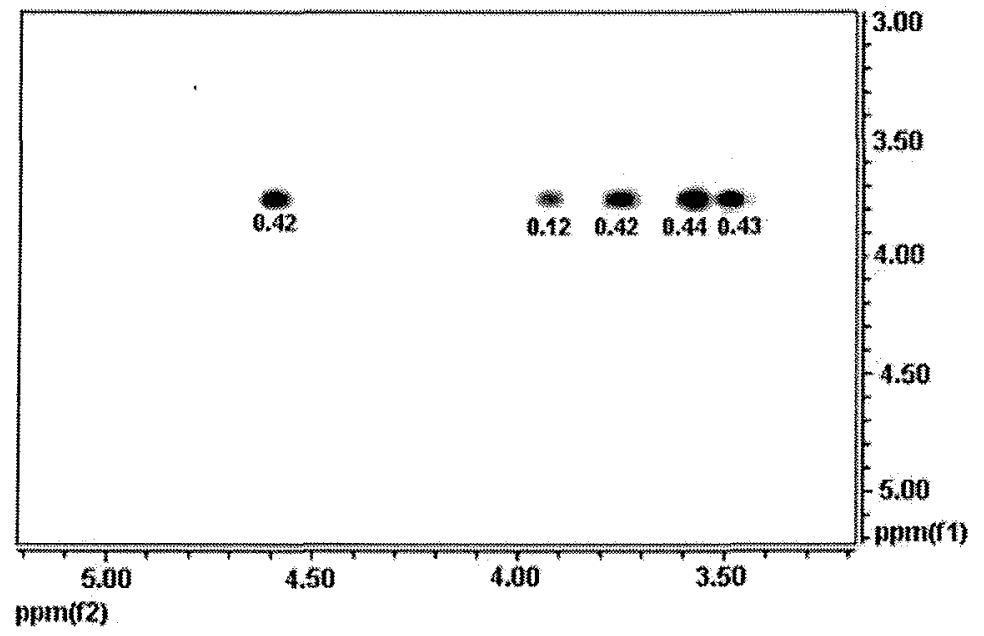

Fig. 2 Calculated TOCSY spectrum for the nuclear spin density matrix $\sigma_{1}=\alpha E+0.7 I_{2 z}$, i.e., only the second proton is polarized while all other protons are totally saturated. Next to peaks their integrals are indicated

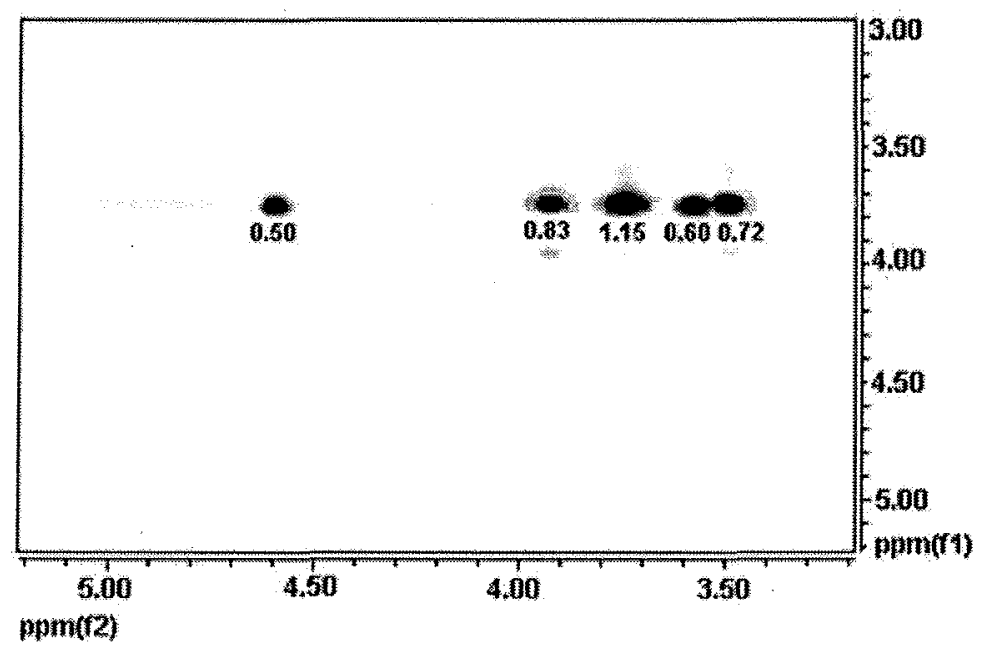

Fig. 3 Calculated TOCSY spectrum for the nuclear spin density matrix $\sigma_{2}=\alpha E+0.7 I_{2 z}+I_{6 z}$, i.e., only the second and the sixth protons are polarized while other protons are totally saturated. Next to peaks their integrals are indicated

matrix $\mathbf{S}$ and calculated vector $\mathbf{I}_{\exp }$ for density matrices $w_{1}$ and $w_{2}$ exactly reconstructed these "unknown" vectors $w_{1,2}$ in both cases.

\section{Conclusions}

A consistent description of STD formation has been given and STD kinetic equations have been obtained which incorporate kinetic rate constants of association and dissociation and the diffusion-controlled rate constant. The effect of spin evolution in free ligand after saturation transfer from the protein to the ligand has been taken into account, which is especially important for strongly coupled nuclei in the saturated ligand. The STD TOCSY. spectra simulation program has been written 
and on its basis a reconstruction procedure of determination of STD nuclear polarizations from TOCSY spectra has been elaborated and it has been shown that initial (STD) saturations can be accurately determined from TOCSY peak intensities by employing the proposed procedure.

Acknowledgments This work was supported by Alexander von Humboldt Stiftung, Russian Foundation for Basic Research Project Nos. 07-03-00424, Program of Division of Chemistry and Material Science, Russian Academy of Sciences Project No. 5.1.1. H.M. thanks the University of Konstanz and the Konstanz Research School-Chemical Biology (KoRS-CB) for continuous support.

\section{References}

1. M. Mayer, B. Meyer, Angew. Chem. Int. Ed. Engl. 111, 1902-1906 (1999)

2. M. Mayer, B. Meyer, J. Am. Chem. Soc. 123, 6108-6117 (2001)

3. B. Meyer, T. Peters, Angew. Chem. Int. Ed. 42, 864-890 (2003)

4. T. Carlomagno, Annu. Rev. Biophys. Biomol. Struct. 34, 245-266 (2005)

5. J. Angulo, C. Rademacher, T. Biet, A.J. Benie, A. Blume, H. Peters, M. Palcic, F. Parra, T. Peters, Meth. Enzymol. 416, 12-30 (2006)

6. C. Rademacher, J. Landstrom, N. Sindhuwinata, M.M. Palcic, G. Widmalm, T. Peters, Glycoconj. J. 27, 349-358 (2010)

7. M.A. Oberli, M. Tamborrini, Y.H. Tsai, D.B. Werz, T. Horlacher, A. Adibekian, D. Gauss, H.M. Möller, G. Pluschke, P.H. Seeberger, J. Am. Chem. Soc. 132, 10239-10241 (2010)

8. H.M. Möller, N. Serttas, H. Paulsen, J.M. Burchell, J. Taylor-Papadimitriou, B. Meyer, Eur. J. Biochem. 269, 1444-1455 (2002)

9. A. Canales, J. Rodriguez-Salarichs, C. Trigili, L. Nieto, C. Coderch, J.M. Andreu, I. Paterson, J. Jimenez-Barbero, J.F. Diaz, Am. Chem. Soc. Chem. Biol. 6(8), 789-799 (2011)

10. V. Jayalakshmi, N.R. Krishna, J. Am. Chem. Soc. 127, 14080-14086 (2005)

11. M. Mayer, T.L. James, J. Am. Chem. Soc. 124, 13376-13377 (2002)

12. R. Meinecke, B. Meyer, J. Med. Chem. 44, 3059-3065 (2001)

13. A.J. Benie, R. Moser, E. Bauml, D. Blaas, T. Peters, J. Am. Chem. Soc. 125, 14-15 (2003)

14. B. Claasen, M. Axmann, R. Meinecke, B. Meyer, J. Am. Chem. Soc. 127, 916-919 (2005)

15. C.A. Lepre, J.M. Moore, J.W. Peng, Chem. Rev. 104(8), 3641-3676 (2004)

16. M. Pellecchia, I. Bertini, D. Cowburn, C. Dalvit, E. Giralt, W. Jahnke, T.L. James, S.W. Homans, H. Kessler, C. Luchinat, B. Meyer, H. Oschkinat, J. Peng, H. Schwalbe, G. Siegal, Nat. Rev. Drug Disc. 7, 738-745 (2008)

17. F.E. Koehn, Prog. Drug Res. 65, 177-210 (2008)

18. C. Dalvit, P. Pevarello, M. Tato, M. Veronesi, A. Vulpetti, M. Sundstrom, J. Biomol. NMR 18, 65-68 (2000)

19. C. Ludwig, P.J. Michiels, X. Wu, K.L. Kavanagh, E. Pilka, A. Jansson, U. Oppermann, U.L. Guenther, J. Med. Chem. 51, 1-3 (2008)

20. J. Yan, A.D. Kline, H. Mo, E.R. Zartler, M.J. Shapiro, J. Am. Chem. Soc. 124, 9984-9985 (2002)

21. V.M. Sanchez-Pedregal, M. Reese, J. Meiler, M.J. Blommers, C. Griesinger, T. Carlomagno, Angew. Chem. Int. Ed. Engl. 44, 4172-4175 (2005)

22. R. Auer, K. Kloiber, A. Vavrinska, L. Geist, N. Coudevylle, R. Konrat, J. Am. Chem. Soc. 132, 1480-1481 (2010)

23. J. Yan, A.D. Kline, H. Mo, M.J. Shapiro, E.R. Zartler, J. Magn. Reson. 163, 270-276 (2003)

24. S. Kemper, M.K. Patel, J.C. Errey, B.G. Davis, J.A. Jones, T.D. Claridge, J. Magn. Reson. 203, 1-10 (2010)

25. V. Jayalakshmi, N.R. Krishna, J. Magn. Reson. 155, 106-118 (2002)

26. V. Jayalakshmi, T. Biet, T. Peters, N.R. Krishna, J. Am. Chem. Soc. 126, 8610-8611 (2004)

27. V. Jayalakshmi, N.R. Krishna, J. Magn. Reson. 168, $36-45$ (2004)

28. N.R. Krishna, V. Jayalakshmi, Progr. Nucl. Magn. Reson. Spectrosc. 49, 1-25 (2006)

29. M.A. Johnson, B.M. Pinto, J. Am. Chem. Soc. 124, 15368-15374 (2002) 
30. M. Rinnbauer, B. Ernst, B. Wagner, J. Magnani, A. Benie, T. Peters, Glycobiology 13, 435-443 (2003)

31. O.G. Berg, Chem. Phys. 31, 47-57 (1978)

32. S.A. Rice, in Diffusion-Limited Reactions, Comprehensive Chemical Kinetics, vol. 25, ed. by C.H. Bamford, C.F.H. Tipper, R.G. Compton (Elsevier Science, Amsterdam, 1985)

33. K.L. Ivanov, N.N. Lukzen, J. Chem. Phys. 121, 5109-5114 (2004)

34. V.M. Berdnikov, A.B. Doktorov, Chem. Phys. 69, 205-212 (1982)

35. V.P. Sakun, Physica 80A, 128-148 (1975)

36. A.B. Doktorov, Physica 90A, 109-136 (1978)

37. K.M. Salikhov, Yu.N. Molin, R.Z. Sagdeev, A.L. Buchachenko, in Spin Polarization and Magnetic Effects in Radical Reactions (Elsevier, Amsterdam, 1984)

38. D.F. Calef, J.M. Deutch, Annu. Rev. Phys. Chem. 34, 493-524 (1983) 